Laser Chem., Vol. 16, pp. 151-156

Reprints available directly from the publisher

Photocopying permitted by license only
(C) 1996 OPA (Overseas Publishers Association) Amsterdam B.V. Published in The Netherlands by Harwood Academic Publishers GmbH

\title{
HIGH-RESOLUTION NON-RESONANT TWO-PHOTON THRESHOLD PHOTOIONIZATION OF PROPYNE
}

\author{
HIROSHI MATSUI, YI-FEI ZHU, and EDWARD R. GRANT \\ Department of Chemistry, Purdue University, \\ West Lafayette, Indiana 47906, USA
}

(Received 27 March, 1995)

\begin{abstract}
Using techniques of delayed pulsed-field ionization zero-kinetic-energy (ZEKE) photoelectron spectroscopy, we have resolved spin-orbit structure in the rotational profile of the origin band in the non-resonant two-photon threshold photoionization of propyne. Both the rotational and spin-orbit characteristics of this band are well-simulated by a simple model that assumes the spin-orbit splitting of acetylene cation in combination with the rotational constants of the neutral propyne ground state. This result, combined with very little evidence for photoionizing transitions terminating on vibronically excited states, suggests that the structure of the propyne cation structure is very close to that of the neutral ground state and very little altered by Jahn-Teller distortion.
\end{abstract}

KEY WORDS: Photoelectron, Laser, Spectroscopy, Propyne, Two-photon, Ionization.

\section{INTRODUCTION}

The widening use of methods for high-resolution threshold photoionization employing zero-kinetic-energy (ZEKE) photoelectron detection has opened a revealing new window on photoionization dynamics and cation structure. ${ }^{1}$ ZEKE techniques exploit a distinctive means for registering state-to-state threshold ionization that relies on the extraordinarily long lifetimes of very-high Rydberg states created in the presence of weak electrostatic fields. ${ }^{2}$ Delayed extraction under these conditions discriminates against the background of other electrons produced by direct photoionization and prompt autoionization, to yield sharp resonances at the ionization limit associated with each cation internal state, $\mathbf{M}^{+}(\mathrm{T}, \mathrm{v}, \mathrm{J})$. With care, one can combine similar delayed pulsed field techniques with cation detection to achieve comparable resolution with mass identification. ${ }^{3}$

Simple alkynes are widely encountered as intermediates in combustion and as reactants in number of chemical processes. Photoionization spectroscopy has long provided information of importance on bond strengths and heats of formation for these and other hydrocarbons. Now, high-resolution threshold photoelectron spectroscopy is providing a much more detailed view of ionization limits and cation structure for an increasing number of key open shell hydrocarbon cations. Among the simplest of these, $\mathrm{C}_{2} \mathrm{H}_{2}^{+}$, has been thoroughly characterized by threshold photoionization, ${ }^{4}$ and by comparison with the ion core structure of non-penetrating Rydberg states. ${ }^{5}$ Propyne 
represents an important step in complexity, adding the simplest of alkyl substituents to the carbon-carbon triple bond. The frontier electronic structure is still dominated by the pi-orbitals of the triple bond, so as with acetylene, ionization of propyne produces an open-shell electronically degenerate cation.

For polyatomic molecules, coupling between vibrational and electronic angular momentum breaks electronic degeneracies, and stabilizes distorted vibronic configurations. In the ${ }^{2} \Pi$ ground state of acetylene cation, this interaction manifests itself in a Renner-Teller coupling which links orbital motion of the unpaired $\pi$ electron with $\mathrm{H}-\mathrm{C}-\mathrm{C}-\mathrm{H}$ trans-bending vibration. In propyne, the presence of off-axis hydrogens introduces the formal possibility of lower-order, linear (Jahn-Teller) coupling terms, because a non-linear molecule always possesses at least one non-totally symmetric normal coordinate that can couple components of the degenerate electronic wavefunction in first order.

In both molecules, spin-orbit coupling further breaks the electronic degeneracy to form states of the double group, in which the pairs of electronic terms linked by vibronic coupling are separated by the spin-orbit splitting. ${ }^{6} \mathrm{By}$ this splitting, spin-orbit coupling tends to quench vibronic coupling. Thus, acetylene cation exhibits observable spin-orbit coupling $\left(30.91 \mathrm{~cm}^{-1}\right)$, with moderate Renner-Teller vibronic coupling $\left(\varepsilon_{4}=0.3, \varepsilon_{4} \omega_{4}=244 \mathrm{~cm}^{-1}\right)$. In quasi-linear propyne cation, the carbon nuclear charge dictates comparable spin-orbit coupling, but the question remains as to the impact of such splitting as there may be on Jahn-Teller coupling. In the present work, we apply threshold photoionization to explore the issue of comparative spin-orbit and vibronic coupling in propyne. We find rovibronic structure that suggests spin-orbit splitting comparable to that of acetylene in concert with very weak Jahn-Teller coupling.

\section{EXPERIMENT}

Experiments investigating the high-resolution threshold photoionization of propyne employ standard methods of non-resonant two-photon ZEKE photoelectron spectroscopy. ${ }^{7}$ The apparatus has been described previously. ${ }^{8}$ Briefly, a skimmed molecular beam enters a magnetically shielded longitudinally oriented ZEKE spectrometer through a grid in the first plate of a three-element ion-optics assembly, comprised of a repeller-extractor pair, spaced by about $1.5 \mathrm{~cm}$, followed $2 \mathrm{~mm}$ further by an entrance grid. The entrance grid caps a grounded $25 \mathrm{~cm}$ field-free flight tube that ends with a final grid isolating a two-element multichannel plate detector. Threshold electrons are produced by two-photon absorption of dye laser radiation (Lambda Physik EMG 202 $\mathrm{MSC} / \mathrm{F} 1$ 2002) frequency-doubled in BBO. Visible output using Coumarin 480 is 10 $\mathrm{mJ} /$ pulse. Ultraviolet light is focussed into the spectrometer by a $30 \mathrm{~cm}$ focal length lens.

Ions and ZEKE Rydberg states are produced between the repeller and extractor in a DC field of $1.6 \mathrm{~V} / \mathrm{cm}$, which sweeps away background electrons produced by prompt ionization channels. The dye laser is scanned, and a pulsed field of $4 \mathrm{~V} / \mathrm{cm}$, applied with a delay of $3.8 \mathrm{~ms}$ following each laser pulse, field ionizes long-lived high principal quantum number Rydberg states which are present whenever the laser frequency falls within a few wavenumbers of the ionization threshold corresponding to each accessible 
cation internal state. Reversing the bias on the detector, we can detect cations following extraction in a field of $200 \mathrm{~V} / \mathrm{cm}$. A Burleigh Model 4500 pulsed wavemeter calibrates laser frequencies which are corrected to vacuum wavenumbers.

\section{RESULTS}

Figure 1 shows a spectrum taken in the doubled dye-laser region of $\sim 42000 \mathrm{~cm}^{-1}$ with cation detection. The features present in this spectrum emerge only at relatively high focussed laser powers, which indicates that the resonance lies at the level of the second photon. A two-photon energy of $84000 \mathrm{~cm}^{-1}$ falls near the estimated ionization potential of propyne, ${ }^{9}$ which suggests that the observed increase in $\mathrm{CH}_{3} \mathrm{CCH}^{+}$signal above $83000 \mathrm{~cm}^{-1}$ approximately marks the ionization threshold.

A scan of the threshold photoelectron spectrum confirms this suggestion. The inset of Figure 2 shows the ZEKE photoelectron signal obtained following two-photon excitation using delayed pulsed-field discrimination. The spectrum of this signal shows one prominent peak at the position of the threshold in the cation signal. This signal is strongest in relation to the background for the higher sample density of a neat propyne expansion. Under those conditions a pair of ZEKE resonances of declining intensity emerge to the red of the singular low-density threshold, as shown in the main part of

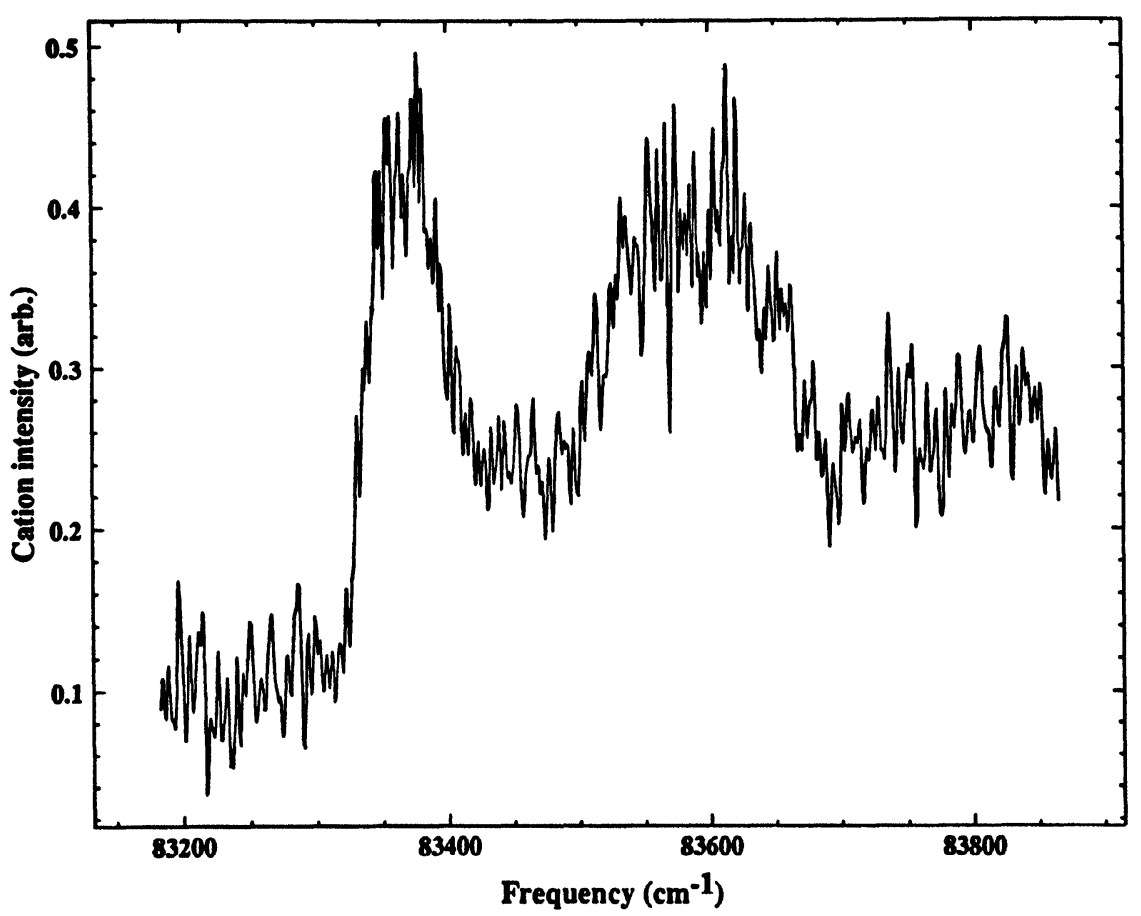

Figure 1 Non-resonant two-photon photoionization spectrum of propyne detected near its $10.3 \mathrm{eV}$ ionization threshold obtained by collecting the $\mathrm{CH}_{3} \mathrm{CCH}^{+}$signal as a function of the excitation frequency. 
Figure 2. We associate these features lying to the red of the threshold resonance with molecular dimers and clusters formed under high-density expansion conditions. No assignable structure can be discerned to the blue of the origin.

\section{DISCUSSION}

Two-photon ionization of propyne with ZEKE photoelectron discrimination yields a spectrum showing distinct structure in the region of the adiabatic ionization potential. In accord with previous observations of nonresonant two-photon ZEKE spectra, including that of acetylene, we associate these features with the structure of the internal states of propyne accessible in threshold photoionization. The electronic structure of propyne is $\left(1 a_{1}\right)^{2}\left(2 a_{1}\right)^{2}(1 e)^{4}\left(3 a_{1}\right)^{2}\left(4 a_{1}\right)^{2}(2 e)^{4}\left(5 a_{1}\right)^{2}(3 e)^{4}$. Thus, threshold ionization produces an e vacancy, giving rise to $\mathrm{a}^{2} \mathrm{E}$ term. Spin orbit coupling produces substates ${ }^{2} E_{1 / 2}$ and ${ }^{2} E_{3 / 2}$ analogous to the ${ }^{2} \Pi$ states of acetylene.

While splitting characteristic of the carbon nuclear mass can be expected, this may be moderated by the quasi-linear geometry of propyne. Another significant consequence of methyl substitution is a small but finite moment of inertia about the $\mathrm{C}-\mathrm{C}-\mathrm{C}$

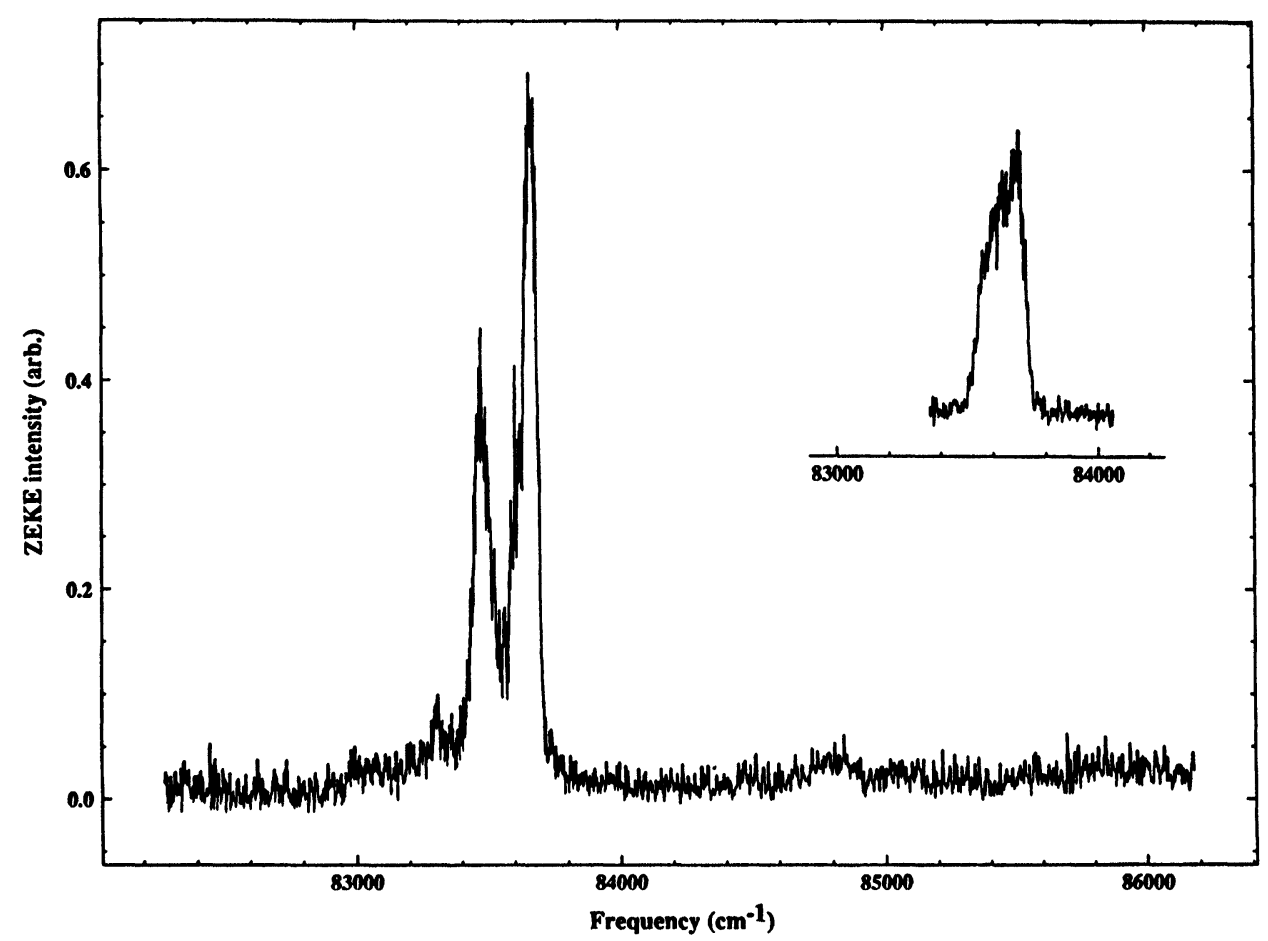

Figure 2 Delayed pulsed-field threshold ionization zero-kinetic-energy photoelectron spectrum of propyne taken under mild expansion conditions-diluted in Ar, $1 \mathrm{~atm}$ stagnation pressure (inset), and in a more concentrated neat expansion. Structure appearing to the red of the most intense band in the main spectrum is assigned to the threshold ionization of propyne-propyne complexes formed under denser expansion conditions. 
internuclear axis, which gives rise to vibronic transitions with the broad sub-structure characteristic of a large rotational constant. We proceed now to attempt to explain in these terms the overall rotational profile of the band that we label as the origin.

As a first approximation we take the spin-orbit splitting characteristic of carbon as observed in acetylene. To compare with the observed rotational profile we then can most simply incorporate this splitting in a Hund's case (b) hamiltonian, choosing rotational constants for the cation that are the same as those well known for ground-state neutral propyne. ${ }^{10}$ Using rotational selection rules for two-photon absorption, and considering transitions only to the totally symmetric continuum, we find that this highly simplified model produces a simulated threshold photoionization spectrum which agrees quite well with that observed experimentally, as shown in Figure 3.
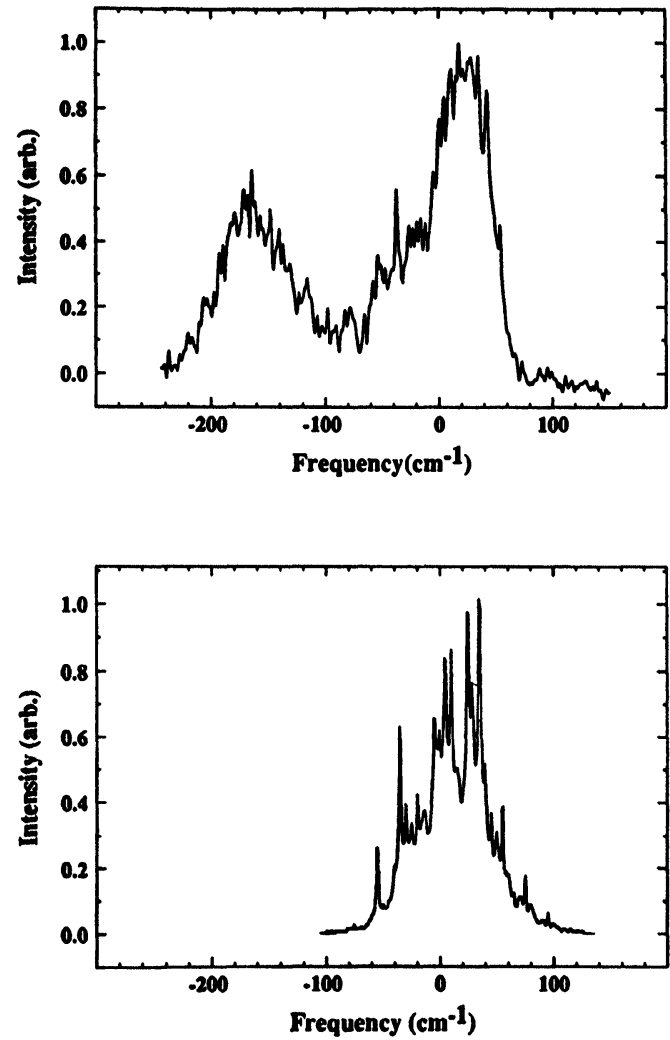

Figure 3 (upper) Expanded view of threshold photoionization structure assigned to production of the cation vibrational ground state. (lower) Simulation of the rotational profile for two-photon photoionization to the totally symmetric continuum, assuming $\mathrm{CH}_{3} \mathrm{CCH}^{+}$cation rotational constants unchanged from those of the neutral ground state and a spin orbit splitting in the propyne cation of $30 \mathrm{~cm}^{-1}$, matching that found for acetylene. 
The strength of these bands in comparison with any other discernible structure in the spectrum further affirms that the cation geometry is similar to that of the neutral molecule. Above the origin, no intensity can be seen in transitions to cation vibrational states excited in the lowest e modes, $\mathrm{C}-\mathrm{C} \equiv \mathrm{C}$ bend $\left(\mathrm{v}_{10}\right.$, neutral ground state frequency $\left.327.5 \mathrm{~cm}^{-1}\right), \mathrm{C} \equiv \mathrm{C}-\mathrm{H}$ bend $\left(\mathrm{v}_{9}, 633.3 \mathrm{~cm}^{-1}\right)$ and methyl skeletal rock $\left(\mathrm{v}_{8}\right.$, $\left.1036.1 \mathrm{~cm}^{-1}\right)$. Nor are transitions seen in totally symmetric modes, such as $\mathrm{C}-\mathrm{C}$ stretch $\left(\mathrm{v}_{5}, 930.3 \mathrm{~cm}^{-1}\right)$. This is consistant with the idea that the structure of the molecule changes little with electron ejection. The absence of intensity in transitions to low-frequency E excited states establishes in particular that vibronic activity and distortion in these coordinates if present is very weak.

\section{CONCLUSIONS}

We have resolved spin-orbit structure in the rotational profile of the origin band in the non-resonant two-photon threshold photoionization of propyne. Both the rotational and spin-orbit characteristics of this band are well simulated by a simple model that assumes the spin-orbit splitting of acetylene cation in combination with the rotational constants of the neutral ground state. This result, combined with very little evidence for photoionizing transitions terminating on vibronically excited states, suggests that the structure of the propyne cation structure is very close to that of the neutral ground state and very little altered by Jahn-Teller distortion.

\section{ACKNOWLEDGEMENTS}

This work was supported by the U.S. Department of Energy under grant No. DEFG02-93ER14401.

\section{References}

1. K. Müller-Dethlefs and E. W. Schlag, Ann. Rev. Phys. Chem., 42, 109 (1991); W. Habenicht, G. Reiser and K. Müller-Dethlefs, J. Chem. Phys., 95, 4809 (1991).

2. W. A. Chupka, J. Chem. Phys., 98, 4520(1993); ibid,99, 5800 (1993); L. Ya. Baranov, R. Kris, R. D. Levine and U. Even, J. Chem. Phys., 100, 186(1994); F. Merkt and R. N. Zare, J. Chem. Phys., 101, 3495 (1994); M. J. J. Vrakking and Y. T. Lee, manuscript submitted (1995).

3. L. Zhu and P. Johnson, J. Chem. Phys.,94, 5769 (1991); H. Krause and H. J. Neusser, J. Chem. Phys., 97, 5923 (1992); H. J. Dietrich, R. Lindner and K. Müller-Dethlefs, J. Chem. Phys., 101, 3399 (1994).

4. S. T. Pratt, P. M. Dehmer, J. L. Dehmer, J. Chem. Phys., 99, 6233 (1993).

5. Y. F. Zhu, R. Shehadeh and E. R. Grant, J. Chem. Phys., 99, 5723 (1993).

6. G. Herberg, Electronic Spectra of Polyatomic Molecules (Van Nostrand Reinhold, New York, 1966) pp 53f; F. T. Chau and L. Karlsson, Phys. Scr., 16(248), (1977); J. T. Hougen, J. Mol. Spectros., 81, 73 (1980).

7. I. Fischer, A. Strobel, J. Staecker, G. Niedner-Schatteburg, K. Müller-Dethlefs and V. E. Bondybey, J. Chem. Phys., 96, 7171 (1992).

8. Yi-Fei Zhu and Edward R. Grant, J. Phys. Chem., 97, 9582 (1993); G. P. Bryant, Y. Jiang, M. Martin, and E. R. Grant, J. Chem. Phys., 101, 7199 (1994).

9. S. G. Lias, J. E. Bartmess, J. F. Liebman, J. L. Holmes, R. D. Levin and W. G. Mallard, J. Phys. Chem. Ref. Data, 17, Supp. 1 (1988).

10. R. K. Thomas and H. W. Thompson, Spectrochimica Acta, 24A, 1337 (1968); A. Dubrulle, D. Boucher, J. Burie and J. Demaison, J. Mol. Spectros., 72, 158 (1978). 